\title{
Rational Design of Pentamode Metamaterials by Topology Optimization
}

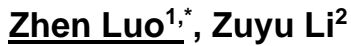 \\ ${ }^{1}$ University of Technology Sydney, Ultimo, 2007, Australia \\ ${ }^{2}$ University of Technology Sydney, Ultimo, 2007, Australia
}

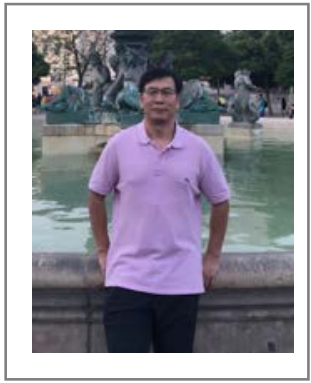

\section{Biography}

Dr Zhen (Jeff) Luo, is currently the Assocate Professor at the Mechanical Engineering, University of Technology Sydney, and he is the director of the JCR Centre “Advanced Digital Design \& Manufacturing”. Dr Luo's current research is focused on advanced lattice metamaterial and the associated multicellular metacomposite technologies using innovative rational design methodologies (e.g. topological optimization) and additive manufacturing techniques, as a generative design-manufacturing-test-characterization integrated system. He has been the author for 112 top tier international journal papers (2019 JCR Q1) since his Ph.D. in 2005, with Scopus citations over 3000 times and h-index 32. He served as organizing committee, mini-symposium organizer, Session Chair, and keynote speaker for 12 prime international conferences. He has been invited to give over 30 seminars, keynotes, guest lectures and talks in international workshops and conference symposia. Dr Luo holds membership of 4 professional organizations, e.g. Int Assoc Comput Mech (IACM), Int Soc Struct Multidiscip Optim (ISSMO), and the Amer Soc Mech Eng (ASME). He has been acted as an assessor for a range of competitive government grants, e.g. ARC, NSFC, and European country foundations (i.e. Ireland, Netherlands, Belgium, Poland, and Croatia). He is an editor for 4 international journals including the flagship journal: Structural and Multidisciplinary Optimization (IF 4.105, JCR-Q1). He has been the principal supervisor for the completion of $8 \mathrm{PhD}$ students. As a Core Investigator he received over 15 research grants including UTS Chancellor's Postdoctoral Fellowship, ARC Discovery Project (DP), Australian AutoCRC, and National Natural-Science-Foundation of China (NSFC), as well as a range of industrial projects.

\section{Graphical Abstract}

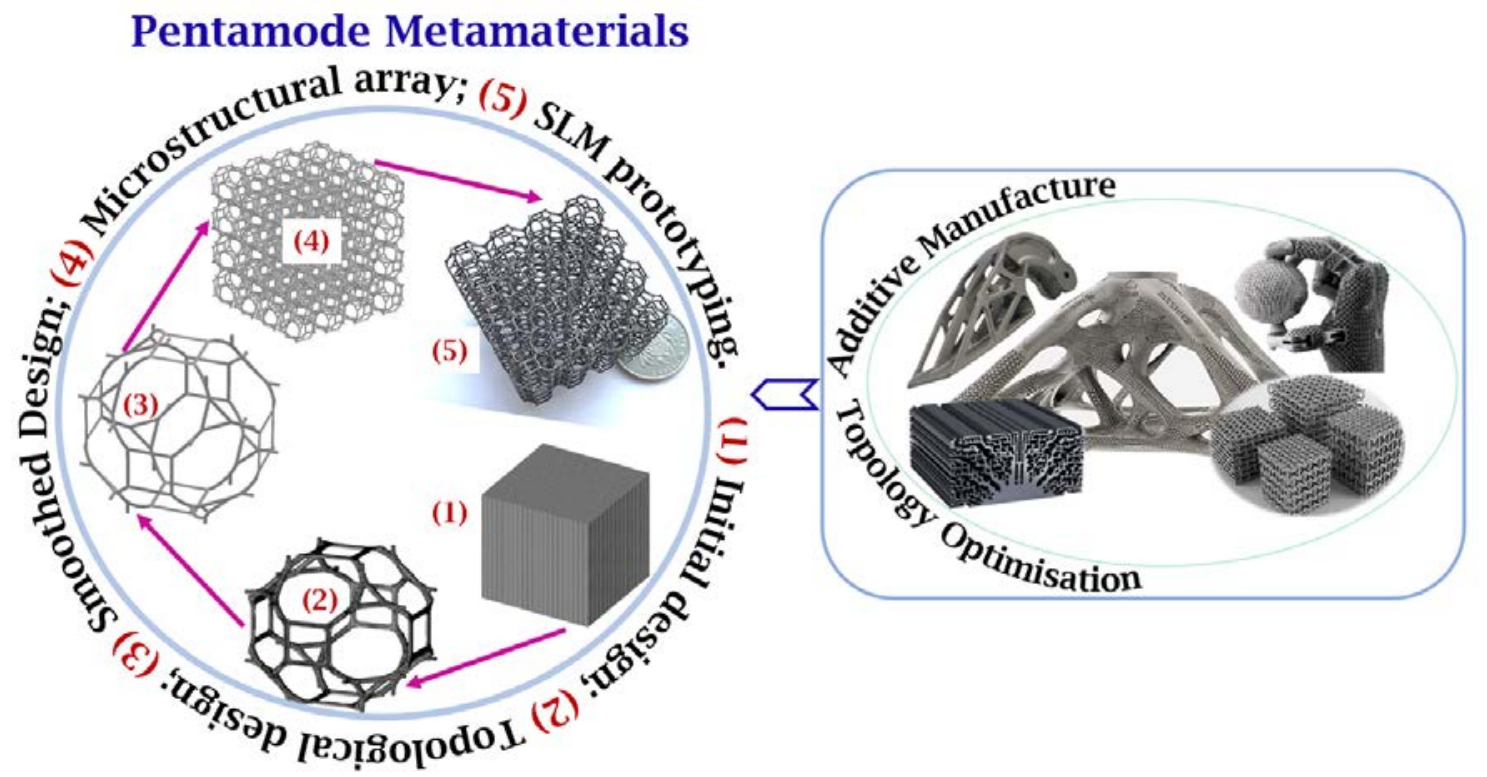

\begin{abstract}
Pentamode metamaterials (PMMs)[1,2], categorized as a new class of three-dimensional solid mechanical metamaterials, refer to artificially engineered lattice materials exhibiting a vanishing shear modulus, mimicking the behaviour of fluids but are solid, and hard to compress yet easy to deform. Here 'penta' denotes five, referring to only one non-zero but five vanishing eigenvalues in the elasticity tensor of isotropic materials. The extraordinary elastic properties of PMMs are dominantly determined by the geometries (i.e. shapes and topologies) of rationally designed microstructures, instead of the composition of their base materials. Compared to most up-to-date design methods based on conventional rigid-body double-cone concept related to diamond lattice,
\end{abstract}




\section{Advanced Materials Lecture Series}

this lecture is more focused an innovative computational design methodology using topology optimization to uncover new micro architectures with novel geometries over a range of effective properties and relative densities. The overall elastic deformation of the microstructure is utilised to achieve the pentamode properties, rather than making use of the highly localised point-point deformation gained from rigid-body links (tiny tips). The design problem is then formulated to make the microstructure have a large but realistically attainable ratio of effective bulk modulus compared to the shear modulus, corresponding to the isotropic microstructure with the effective Poisson's ratio approaching to 0.5. The larger of the ratio, the better of the PMM solids to simulate liquids. The whole microstructure is discretised with a high-resolution finite element mesh, in order to capture the fine geometric features of the ultralight microstructure in the space. The SIMP-topology optimisation method [3] is firstly applied to find the topologically optimised pentaomode microstructure, and then based on the skeleton of the topological design the size optimisation approach is further used to investigate the impact of the size dimensions to the pentomode properties. The SLM (selective laser melting) technique [4] is used for rapid prototyping fabrication of the optimised microstructures with metal materials. Several numerical cases are used to demonstrate the effectiveness of the proposed optimisation method for creating novel pentamode materials. The computation is conducted using UTS ARCLab high-performance computing clusters. It can be found that the effective Position's ratio of the microstructure gradually close to 0.5 and the ratio of effective bulk modulus with respect to the shear modulus is getting larger, along with the reduce of the size dimension of the beam of the microstructure, although the manufacturing is becoming more difficult.

Keywords: Pentamode Metamaterials; Topology Optimization; Three-dimensional solid microstructures; Additive Manufacturing.

Acknowledgements

This research was supported by the Australian Research Council (ARC) -

Discovery Projects (160102491).

\section{Reference}

1. M. Kadic, T. Bückmann, N. Stenger, M. Thiel, M. Wegener, Applied Physics Letters, 2012, 100(19), 191901.

2. M. Kadic, T. Bückmann, R. Schittny, M. Wegener, New Journal of Physics, 2013, 15(2), 023029.

3. M. P., Bendsøe, O. Sigmund, Topology Optimization: Theory, Methods, and Applications. Springer, 2003.

4. I. Gibson, D. Rosen, \& B. Stucker, B. Additive Manufacturing Technologies. Springer, 2015. 